\title{
SIMULATIONS OF HIGH-CURRENT NUMI MAGNETIC HORN STRIPLINES AT FNAL
}

\section{Taylan Sipahi}

Department of Electrical \& Computer Engineering, Colorado State University, Fort Collins, CO, 80523, USA

Universities Research Association (URA) Visiting Scholar at Fermilab

E-mail: taylan@engr.colostate.edu

\section{Stephen Milton}

Department of Electrical \& Computer Engineering, Colorado State University, Fort Collins, CO, 80523, USA

E-mail: milton@engr.colostate.edu

\section{Sandra Biedron}

Department of Electrical \& Computer Engineering, Colorado State University, Fort Collins, CO, 80523, USA

E-mail: biedron@rams.colostate.edu

\section{James Hylen}

Accelerator Division, Accelerator Systems, Target Systems, Target Science Support Fermilab, Batavia, IL 60510, USA

E-mail: hylen@,fnal.gov

\section{Robert Zwaska}

Accelerator Division, Accelerator Systems, Target Systems

Fermilab, Batavia, IL 60510, USA

E-mail: zwaska@,fnal.gov

\begin{abstract}
Both the NuMI (Neutrinos and the Main Injector) beam line, that has been providing intense neutrino beams for several Fermilab experiments (MINOS, MINERVA, NOVA), and the newly proposed LBNF (Long Baseline Neutrino Facility) beam line which plans to produce the highest power neutrino beam in the world for DUNE (the Deep Underground Neutrino Experiment) need pulsed magnetic horns to focus the mesons which decay to produce the neutrinos. The high-current horn and stripline design has been evolving as NuMI reconfigures for higher beam power and to meet the needs of the LBNF design. In this paper, we present calculations, using the Poisson and ANSYS codes, of the EM interaction of the stripline plates of the NuMI horns at critical stress points. In addition, we give the electrical simulation results using the ANSYS Electric code. These results are being used to support the development of evolving horn stripline designs to handle increased electrical current and higher beam power for NuMI upgrades and for LBNF.
\end{abstract}




\section{ACCELERATOR BASED NEUTRINO PRODUCTION}

Neutrino beams from proton accelerators are produced as follows (Figure 1). First, the protons are accelerated in a typical particle accelerator such as a synchrotron. The energy of the protons, along with the geometry of the beamline determines the energy of the neutrinos. The accelerated protons are extracted from the accelerator and directed on to a target where the protons interact with the target material, producing a large number of secondary pions among other particles. Shaped magnetic fields created by focusing horns are used to select out pions of the preferred charge, and focus them into a collimated beam.

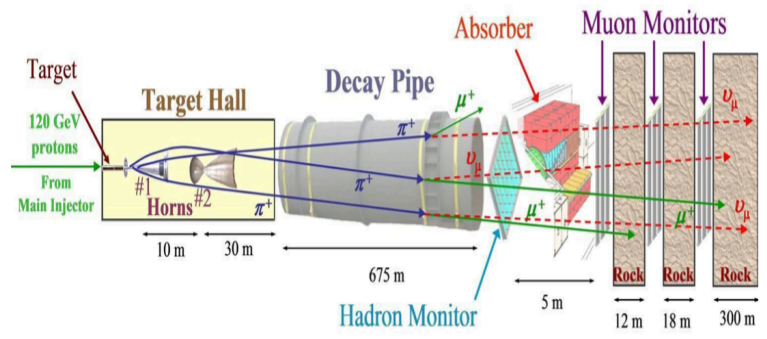

Figure 1. General layout of accelerator based neutrino production [1]

These horns are fed high current through striplines as shown in Figure 2.

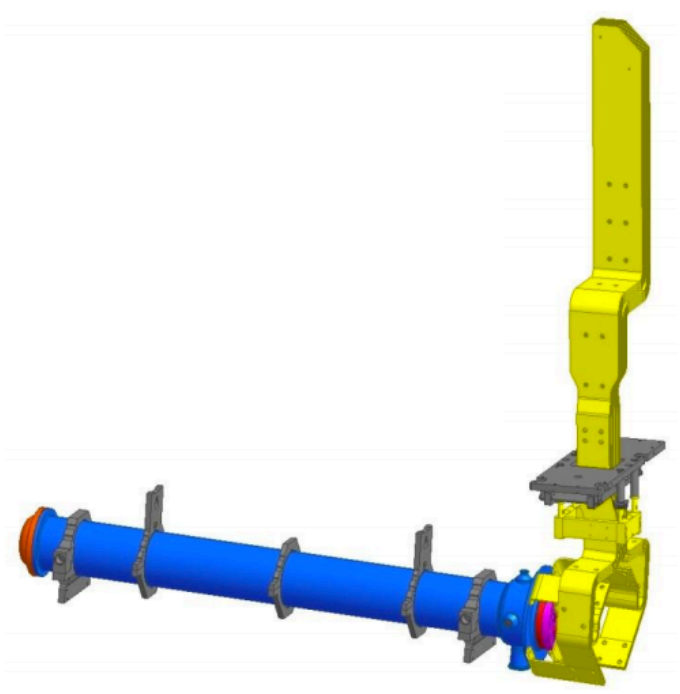

Figure 2. 3D model of the attached magnetic horn (blue section) and the high current stripline (yellow section)

\section{RECENT FERMILAB SPECIFIC HORN SYSTEM CHALLENGES - HIGH-CURRENT STRIPLINES}

Magnetic horns at Fermilab are designed to pulse 10-20 million times a year and are designed near the endurance limit of fatigue. These horns necessarily fail after a few years of operation, the goal being four years. Design of these horns always involve a compromise of various factors which lead to performance or reliability, such as: maximum current, absorption by material, resistive heating, beam-radiation heating, radiation damage, electromechanical forces, thermomechanical forces, cooling, fatigue, erosion, and corrosion.

The stripline shown in Figure 3 has proven itself durable in fatigue resistance and overall robustness, however this stripline was modified, as shown in Figure 4 , to a larger radius to the beam axis, reducing beam heating and increasing air cooling. In comparison to the older design this new design exhibits less symmetry. Also they have been changed to chamfered plates which has three bolt holes in the new design.

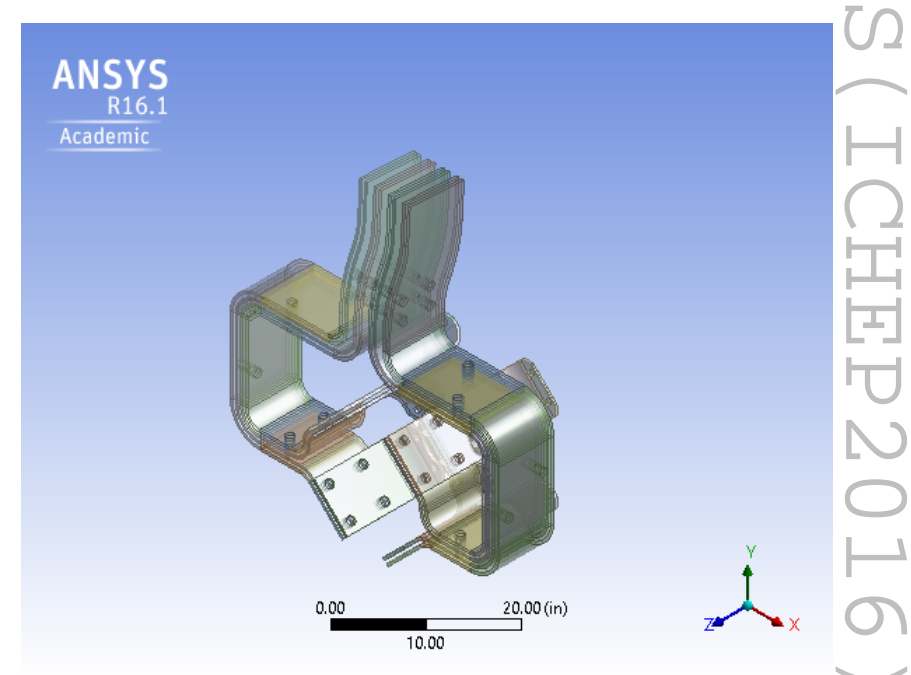

Figure 3. The roboust high current stripline on NuMI/NOvA Horn 1

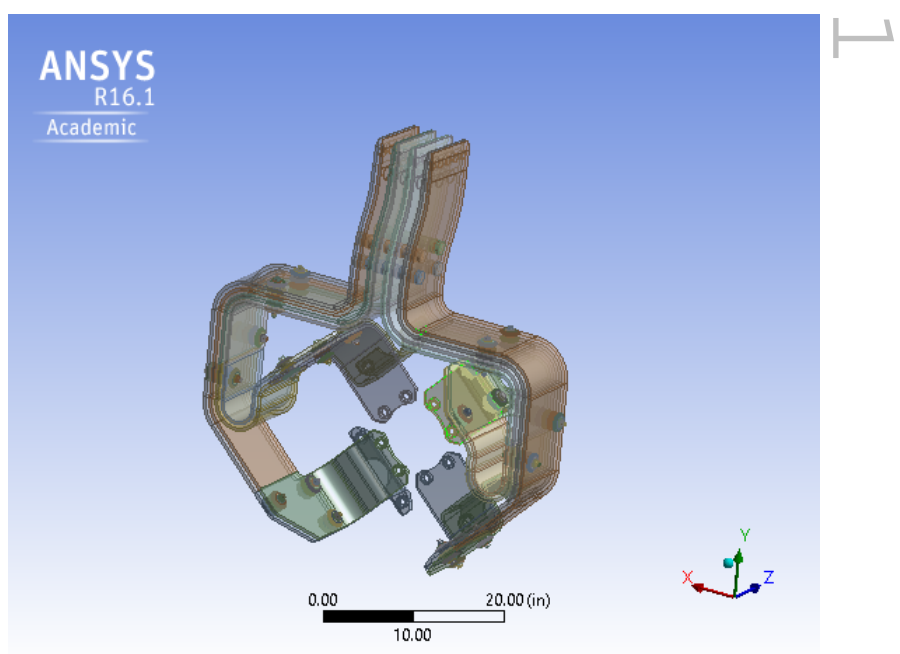

Figure 4. Updated failed high-current stripline on NuMI/NOvA Horn 1

This updated design had a failure due to vibrationenhanced fatigue of an attached stripline. Electromechanical forces from the pulses excited a 424 
$\mathrm{Hz}$ mode on a long span of stripline. This excitation has since been observed to persist for 100 s of pulses per impulse, greatly increasing the number of fatigue cycles the material is subjected to.

Presently, to assist in the engineering design to reach these high currents, we are investigating the electromagnetic details of the magnetic horns and the high-current striplines. From these realistic simulations of the horns and striplines we hope to gain a better understanding of the complete system as well as find potential weaknesses, such as stress-induced structural damage to the striplines and/or the horn structure.

\section{ELECTROMAGNETIC SIMULATIONS OF HIGH-CURRENT STRIPLINES FOR MAGNETIC HORNS}

We have performed simulations of the electromagnetic interaction of the stripline plates of the NuMI/NoVA horn 1 at critical stress points [2, 3, 4], using both the POISSON [5] and ANSYS Maxwell 3D [6] codes for a given applied current of $200 \mathrm{kA}$.

Four conductors are used to conduct the $200 \mathrm{kA}$ into and out of the inner and outer conductor of the horn. The cross section of all plates are 8 inches by 0.375 inches, and the distance between each plate is 0.375 inches. Each carry an applied current of $50 \mathrm{kA}$ pulsed, however during our simulations direct current (DC) case is considered so far.

As an example, two striplines carrying opposing current generate magnetic fields that when coupled to the current generate a repulsive force between the two striplines. With $50 \mathrm{kA}$ of current in each stripline the magnetic force between the two striplines is found to be $6186 \mathrm{~N} / \mathrm{m}$ from POISSON (Figure 5) and $6192 \mathrm{~N} / \mathrm{m}$ as calculated by ANSYS Maxwell 3D (Figure 6).

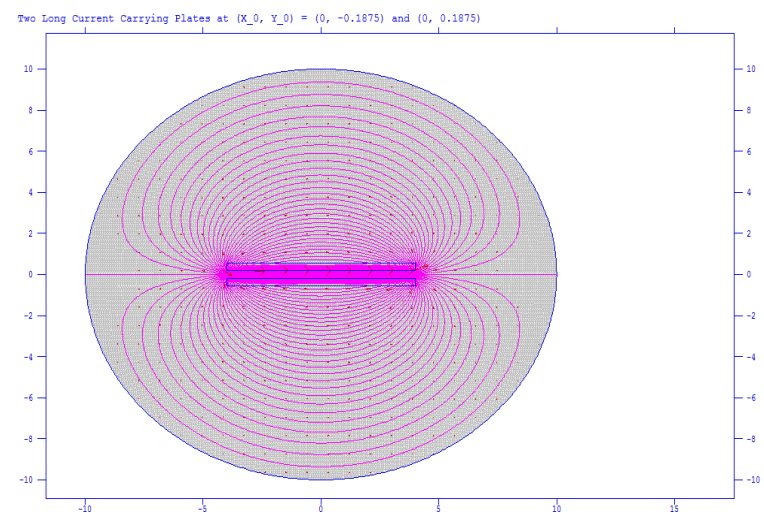

Figure 5. Poisson result for the magnetic field map through a cross section of parallel plates in $\mathrm{x}-\mathrm{y}$ planes each carrying $50 \mathrm{kA}$ DC in the opposite directions

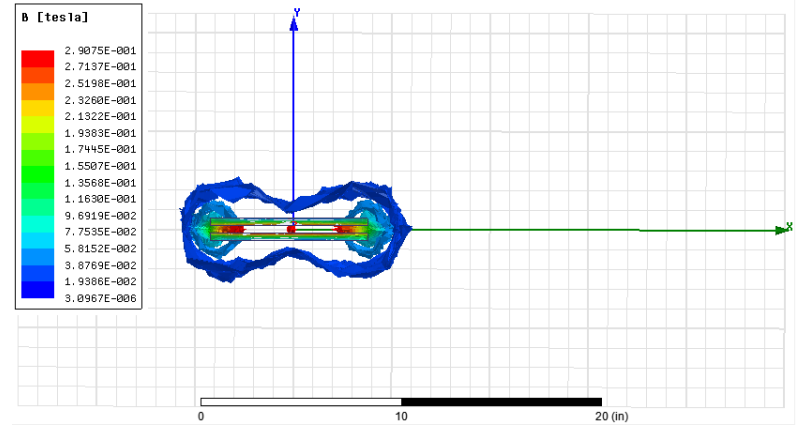

Figure 6. ANSYS Maxwell 3D result for the magnetic field map of two parallel plates of the high-current stripline each carrying $50 \mathrm{kA}$ DC in the opposite directions

After seeing two results are accurate enough for the straight sections of the stripline we continue to simulate using ANSYS Maxwell 3D for the stripline failed in service at the 90 degree bend in the so-called "flag plate" (Figure 7). Simulations were done to see if the magnetic induced stresses were the primary source of the failures at this point.

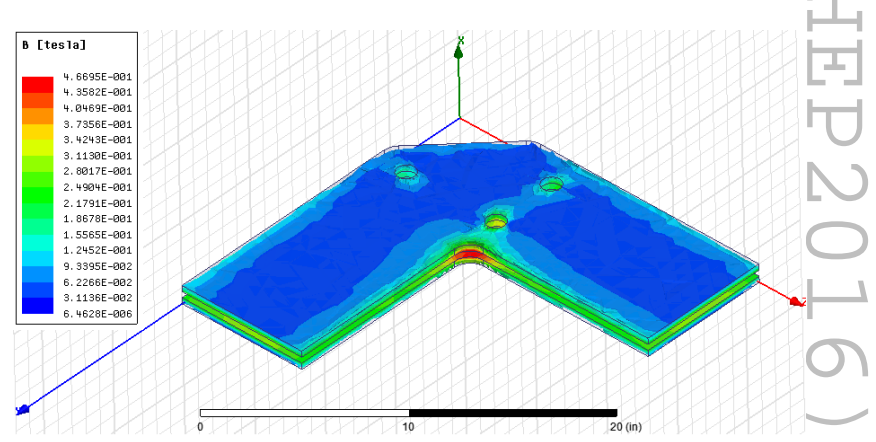

Figure 7. ANSYS Maxwell 3D for the magnetic field distribution of the flage plates of the high-current stripline each carrying $50 \mathrm{kA} \mathrm{DC}$ in the opposite directions

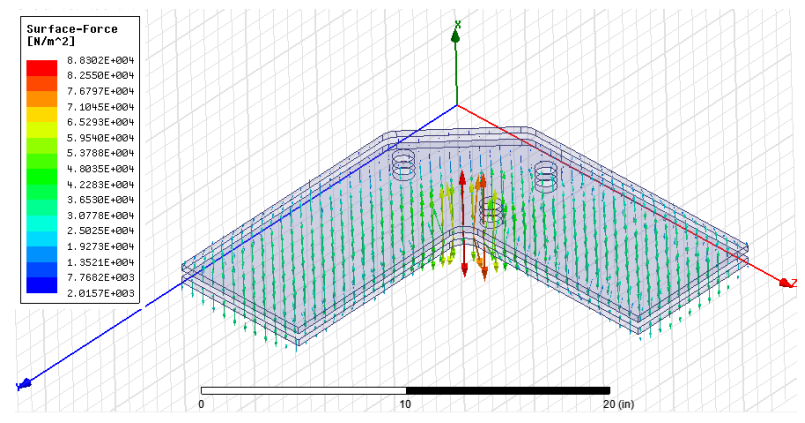

Figure 8. ANSYS Maxwell 3D result for the pressure distribution of the flage plates of the high-current stripline each carrying $50 \mathrm{kA} \mathrm{DC}$ in the opposite directions

Simulations show that the magnetic field at the inner most corner is larger than in the straight sections and the average pressure on the flag plate is found to be $\sim 3.1 \mathrm{x}$ $10^{4} \mathrm{~N} / \mathrm{m}^{2}$; however, at the inner corner section this pressure is $\sim 8.8 \times 10^{4} \mathrm{~N} / \mathrm{m}^{2}$ (Figure 8 ). 


\section{ANSYS ELECTRIC RESULTS FOR CURRENT DENSITY}

The electric current density calculations were performed using the ANSYS Electrical code. $50 \mathrm{kA}$ of current was applied to the flag plate to evaluate the current density in critical stress locations. As a reference point the current density for the simple straight sections is $2.4 \times 10^{6} \mathrm{~A} / \mathrm{ft}^{2}$. When the identical current is applied to the chamfered L-shaped flag plate that includes the bolt holes, the current density at the inner corner is $\sim 3$ times larger than the nominal two straight plates case (Figure 9).

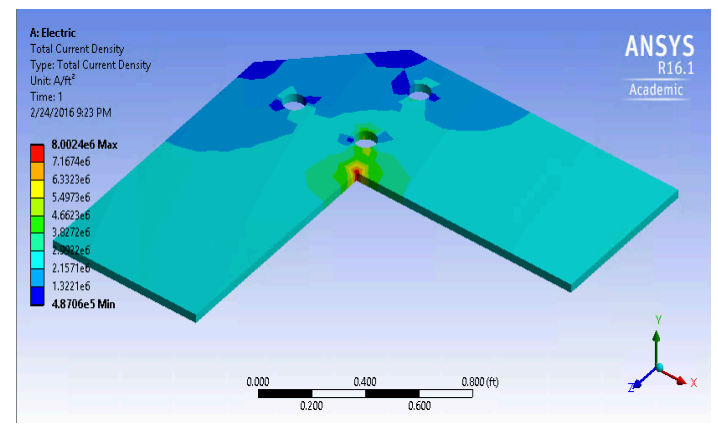

Figure 9. ANSYS Electric result for total current density distribution of the flage plate carrying $50 \mathrm{kA} \mathrm{DC}$

\section{CONCLUSION}

The current densities and forces at the critical locations of the flag plate part were evaluated. Inclusion of the chamfering and bolt holes effected the current density and forces, but not critically. The results for the magnetic field and magnetic force were shown for straight plates. The straight section characteristics agreed well with theory. Additional magnetic field related effects such as the local pressures and current densities were evaluated for real stripline flag plates. The magnetic field was found to be $\sim 2$ times larger at the inner corner of the plates (Figure 7) as compare to the straight plates case (Figure 8). The pressure was found to be $\sim 8.8 \times 10^{4} \mathrm{~N} / \mathrm{m}^{2}$ at the highest point which was $\sim 2.5$ times higher than seen on average shown in Figure 8. The current density is found to be $\sim 3$ times larger in the corners (Figure 9). These results are not high enough to cause the stress fracture and these fractures are more likely related to repetitive heating and/or vibrational effects cause by the current and magnetic forces respectively. These results are being implemented in the on-going design upgrades of the magnetic horn stripline designs to handle increased electrical current and higher beam power for NuMI upgrades and for future LBNF. The corner sections need to be evaluated not only mechanically and thermally but also considering electromagnetic contributions. It is better if the inner corner radius of the plate will be enlarged for the higher current required in the next designs.

\section{ACKNOWLEDGEMENTS}

The work of T. Sipahi is supported by the Visiting Scholars Program of the Universities Research Association (URA) at Fermi National Accelerator Laboratory (FNAL). Fermilab is Operated by FERMI Research Alliance, LLC under Contract No. De-AC0207CH11359 with the U.S. Department of Energy.

\section{REFERENCES}

[1] Yun He, CLASSE Seminar, "Magnetic Focusing Horns for Neutrino Experiments", CHESS, Cornell University, December 17, 2010.

[2] T. Sipahi et al., "Simulations of High Current NuMI Magnetic Horn Striplines at FNAL." in Proc. of the $7^{\text {th }}$ International Particle Accelerator Conference (IPAC'16), Busan, Korea, May 8-13, 2016, paper TUPMR004, (pp. 1230-1233). JACOW, Geneva, Switzerland, 2016.

[3] T. Sipahi et al., "Simulations Of High Current Magnetic Horn Striplines at Fermilab.”, In 2016 North American Particle Accelerator Conference Proceedings NAPAC'16, Chicago, IL, USA, October 9-14, 2016. (pre-press).

[4] T. Sipahi et al., "High Current Striplines for Neutrino Beams." In $6^{\text {th }}$ Euro-Asian Pulsed Power Conference (EAPPC 2016) with the $21^{\text {st }}$ International Conference on High-Power Particle Beams (BEAMS) and the $15^{\text {th }}$ International Conference on Megagauss Magnetic Field Generation and related topics (MEGAGAUSS) Proceedings, Estoril, Portugal, 18-22 September, 2016. (pre-press).

[5] J. Warren, et al., "POISSON/SUPERFISH Reference Manual," Los Alamos National Laboratory report LA-UR-87-126 (1987).

[6] ANSYS Academic Research, Release 16. 